\title{
Recurring Peri-Prosthetic Fracture of the Femur Ultimately Managed by Custom-Made Distal Femoral Mega-Prosthesis - A Case Report and Literature Review
}

\author{
Aftab Younus ${ }^{1}$ and Adrian Kelly ${ }^{2 *}$ \\ ${ }^{1}$ Orthopedic Surgeon, Helen Joseph Hospital, University of the Witwatersrand, Johannesburg, South Africa \\ ${ }^{2}$ Department of Neurosurgery, Dr George Mukhari Academic Hospital, Sefako Makgatho health sciences University, South Africa
}

Submission: May 30, 2020; Published: June 09, 2020

*Corresponding author: Dr. Adrian Kelly, FC Neurosurgery (SA), Dr George Mukhari Academic Hospital, Sefako Makgatho health sciences University, Pretoria, South Africa

\begin{abstract}
Patients with peri-prosthetic fractures of the knee comprise a specific cohort demanding a specific set of orthopedic considerations. These fractures are difficult to manage, and essential orthopedic considerations must include patient factors such as age, co-morbid medical conditions, and overall bone quality. Further fracture considerations go beyond fracture displacement, and if present whether a reduction can be achieved, but must also specifically incorporate unique aspects such as prosthetic alignment, the degree of prosthetic fixation, whether there is a prosthetic stem, distal bone stock, and the extensor mechanism. For ease of understanding peri-prosthetic knee fractures are divided into femoral, tibial, and patellar sub-types, each of which has its own specific epidemiology, classification system, and set of well-defined surgical nuances. Of these three major types peri-prosthetic fractures of the femur are the most common surgical subtype encountered. We present a 65-year old male patient who presented to our unit with a neglected right-sided recurrent peri-prosthetic fracture of his femur, post having undergone multiple surgical interventions at another hospital. Our clinical assessment and X-ray investigation of his right knee revealed what we considered to be profound. With complete loss of distal femoral bone stock, gross malunion, and the complex mass of broken hardware noted, our only option was to remove the distal femur and prosthesis and replace these with a custom-made distal femoral mega-prosthesis. Our case report illustrates an extreme example of a peri-prosthetic fracture of the femur, and we compliment this with a review of the topic in general. It is hoped that our case report and review will assist orthopedic surgeons who perform total knee replacement surgery and will, at some point, need to manage this specific complication.
\end{abstract}

Keywords: Peri-prosthetic knee fractures; Distal femoral mega-prosthesis

\section{Introduction}

The incidence of peri-prosthetic knee fractures is increasing parallel to the increasing incidence of patients who undergo total knee replacement surgery. In terms of quantifying the magnitude of the problem one study from the United States reported an incidence of approximately 300000 cases per year [1]. The distal femur is most commonly involved, and the specific definition provided to define a peri-prosthetic knee fracture at this area is a fracture occurring within $15 \mathrm{~cm}$ of the knee joint line, or within $5 \mathrm{~cm}$ of the intra-medullary implant stem if present [2]. Considering peri-prosthetic knee fractures, specifically considering only those that involve the distal femur, several studies quantify this specific problem and report it to affect between 0.3 and $2.5 \%$ of all knee replacements $[3,4]$. Regarding revision knee replacement surgeries, the incidence escalates considerably and one paper reports this to affect up to $38 \%$ of cases [5]. Peri-prosthetic knee fractures may less commonly involve the proximal tibia which is reported to occur after between $0.3-1.7 \%$ of knee replacement surgeries [6,7].

Peri-prosthetic knee fractures least commonly involve a non-resurfaced patella, where the reported incidence is only $0.05 \%$ [8]. Considering the mechanism of injury most studies report low energy trauma, especially when involving a torsional force, to account for the majority of cases $[9,10]$. The interval between the index knee replacement surgery and the occurrence of a peri-prosthetic femur fracture has been explored in several studies that note the peak incidence to be between 2 and 4 years $[11,12]$. Another study, that again considered only peri-prosthetic femur fractures, reported a mean interval of 70 months [13]. 
These fractures are difficult to manage, and common problems encountered needing specific consideration include poor bone quality, pre-existing bone cement, implant instability, soft tissue adhesions, and vascular damage. How these factors impact outcome is their noted direct association with complications such as non-union, malunion, infection and bone necrosis $[10,14,15]$.

\section{Case Report}

We present a 65-year old male patient who presented to our unit in a wheelchair, unable to ambulate independently for several years, due to what was ultimately diagnosed to be a neglected right-sided peri-prosthetic knee fracture. The surgical history obtained from the patient began 5-years previously where he had undergone staged bilateral total knee replacement surgeries in another hospital. Approximately 2-years after these surgeries he had slipped at home and had incurred his $1^{\text {st }}$ periprosthetic knee fracture involving the right distal femur. At that time, he re-presented to the same hospital where the index surgeries had been performed, and a total right knee revision surgery was performed with stemmed implants. For a year thereafter he was doing well until one day when he again fell, this time while walking down a ramp, and re-fractured the same femur just above the intramedullary stem. This is where he believes his problems really started. He revisited the same hospital where he was taken to surgery and the distal femur was plated. After this 2 nd revision surgery, he noted that although he had an ongoing problem with walking, he was still largely ambulant with a walking stick.

Approximately 6-months later he re-injured himself when he mis-stepped a step, and the same femur fractured for a $3^{\text {rd }}$ time, breaking the plate in the process. He revisited the same hospital, where he was now taken for what was to be his $3^{\text {rd }}$ revision surgery. While he was unsure exactly what was done, he believes a longer plate was simply placed over the broken plate and augmented with 2 wires. Another 6-months passed, during which he had ongoing difficulty ambulating, until he a $4^{\text {th }}$ fall occurred this time on a flat surface inside his house. Although he did not seek medical attention, he knew from previous experience he had re-fractured the same femur. The reason for him not seeking medical attention given was that he was afraid of further surgery which he felt would be of no benefit.

Since that time, for over 2-years until this presentation, he had been battling on his own in considerable pain with significant physical impairment that affected all activities of daily living. What had brought him to the hospital now was a decision that he had made that enough was enough. He had decided to visit our unit as he felt the treatment that he had received at the other hospital had, in his opinion, done more damage than good.

On examination the patient had extensive surgical scars on the distal lateral aspect of the right thigh and anterior surface of the right knee. The right thigh further demonstrated muscle wasting in all compartments as compared to the left. The thigh was visible deformed with 20 degrees of fixed valgus angulation and 10 degrees of external rotation. Palpable callus was present on the medial aspect of the distal right thigh. The right knee had a reduced range of motion with 0-50 degrees of flexion demonstrated. The right lower limb was neurovascularly intact. An $8 \mathrm{~cm}$ limb length discrepancy was also present. An Xray of both knees was performed, and while a standard non-stemmed left knee prosthesis was visualized, what we saw on the right side was unexpected (Figures 1-3).

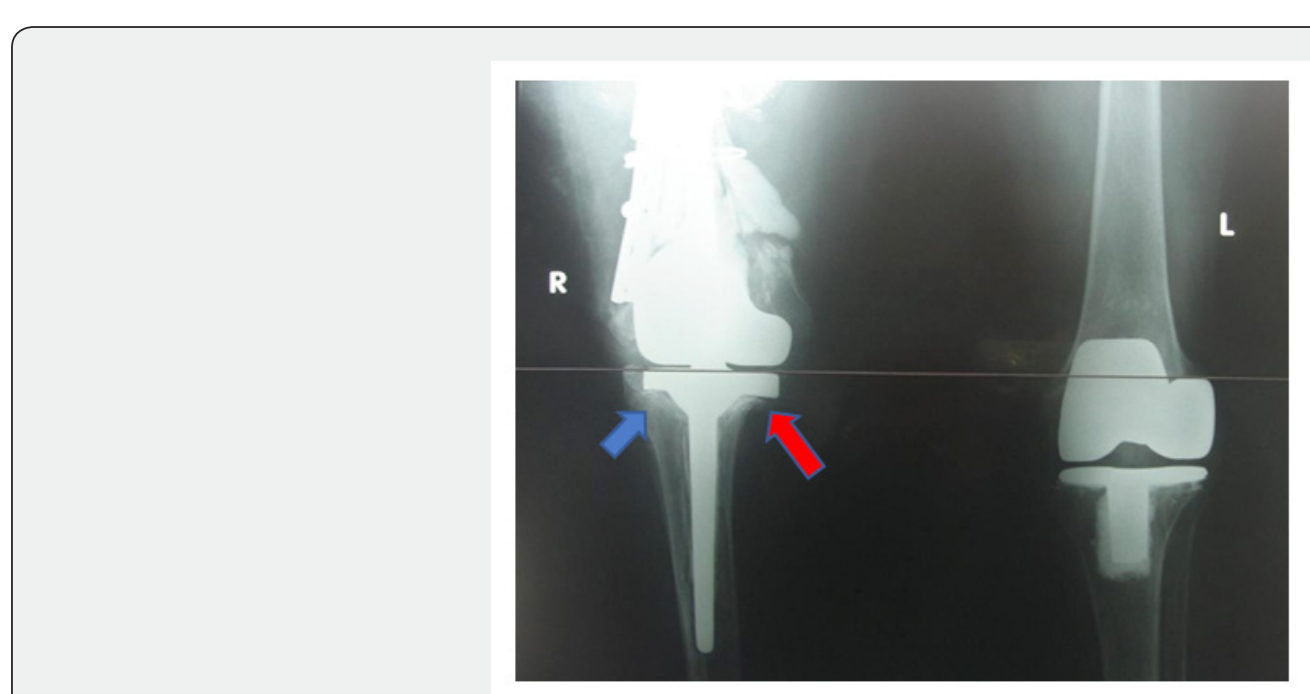

Figure 1: Pre-operative anteroposterior X-rays of both knees. The intact left sided non-stemmed implant is seen, and the stemmed right sided revision implant is seen. With regards the right revision implant the prominence of the medial tibial plateau of the implant (red arrow), compared to the lateral tibial plateau of the implant (blue arrow), is indicative of the 10 degrees of external rotation noted clinically. On this view only a complex, poorly defined, hyperdense mass of hardware is visualized replacing the right distal femur. 


\section{Orthopedics and Rheumatology Open Access Journal (OROAJ)}

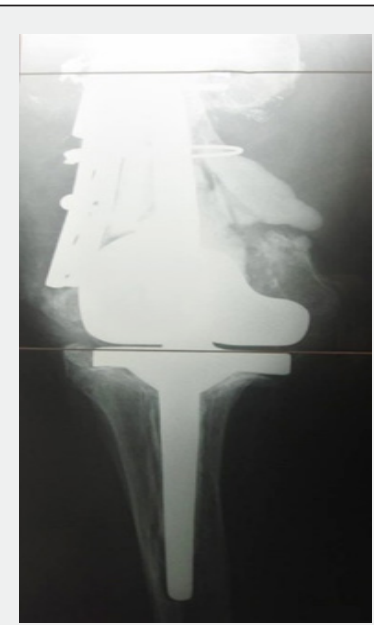

Figure 2: Pre-operative anteroposterior X-ray of the right knee shows profound disuse osteopenia, the same external rotation noted above, and the same complex mass of hardware in in relation to the right distal femur.

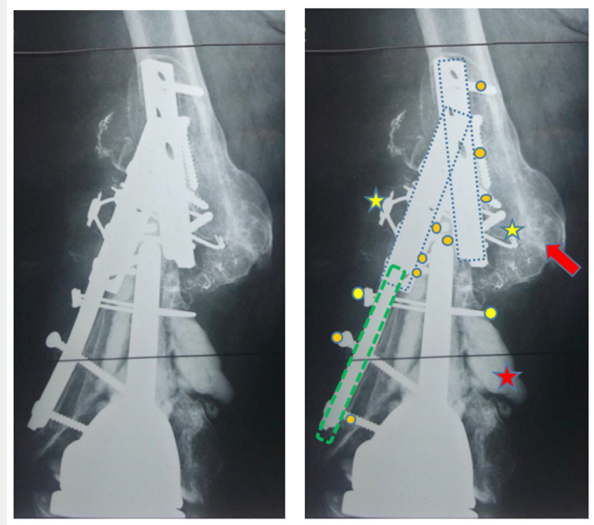

Figure 3: Anteroposterior $\mathrm{X}$-ray of the right distal femur showing a complex mass of hardware in relation to a malunion of a periprosthetic knee fracture involving the distal femur. While difficult to interpret we were able to identify the following, which is indicated in the view on the right.
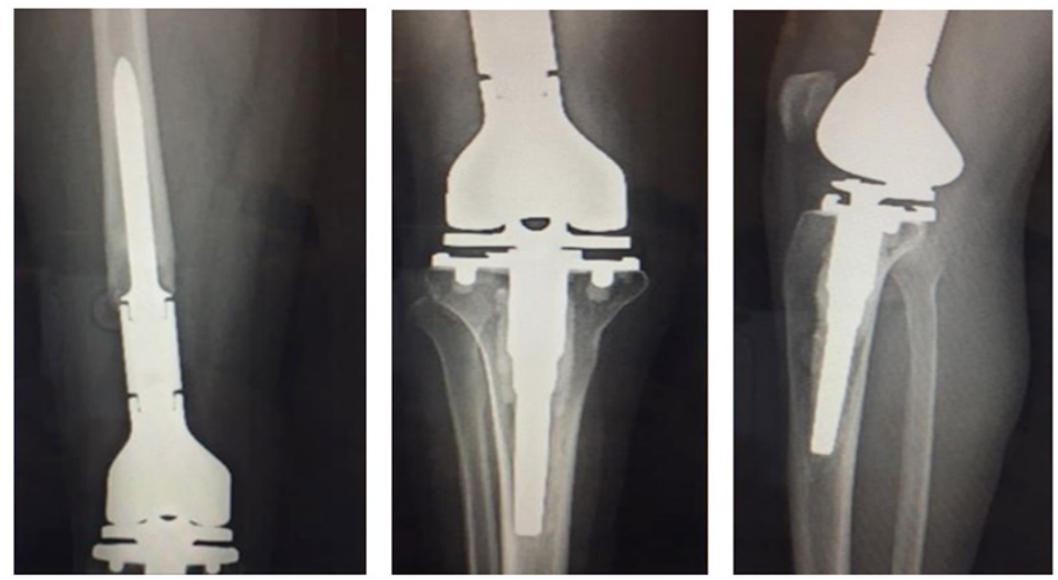

Figure 4: Post-operative anteroposterior and lateral X-rays of the right distal femur and knee showing the custom-made total knee prosthesis.

Figure 4: Post-operative antero-posterior and lateral X-rays of the right distal femur and knee showing the custom made distal femoral mega-prosthesis. 
i. A broken superior cerclage wire can be seen (yellow star), and below it an intact cerclage wire can be seen encircling the lateral plate and femur (yellow circle).

ii. The edges of a broken plate, presumably from the $2^{\text {nd }}$ revision surgery, can be visualized overlapping (blue dotted lines).

iii. A second locking plate can be seen inferior to the $1^{\text {st }}$ (green dotted lines).

iv. Several screws, many of which are not in any bone, can be seen in relation to the hardware mass (orange dots),

v. The rounded edge of the distal right femur can be seen obliterating the medullary cavity (red arrow), and

vi. Free lying bone cement is visualized on the medial side of the femoral stem of the implant (red star).

Due to the significant shortening of the right femur with the distal shaft and femoral condyles essentially destroyed, our only option was to design a custom-made distal femoral megaprosthesis to replace the distal femoral shaft and knee in its entirety.

\section{Surgical Procedure}

Post induction of anesthesia the patient was placed in a supine position on the operating table and a tourniquet was placed and inflated on the upper thigh. A midline incision was commenced in the midline of the distal thigh and was extended distally on the lateral side of the patellar (lateral parapatellar approach) and, in a curvilinear manner, was brought back to the midline of the proximal tibia. The dissection started on the tibial end of this incision, where a lateral subperiosteal dissection sleeve was created, and extended superiorly. The dissection was continued past the prosthetic knee joint on the lateral side of the quadriceps muscle. The subperiosteal dissection was continued superiorly until the entire distal third of the femur was exposed. The patella was then retracted medially. Upon exposure of the distal femoral area a disorganized extensive mass of scarred tissue was noted, which upon dissection contained encased hardware. Only that hardware which was immediately visible was removed. The subperiosteal dissection was extended medially, freeing the entire distal femur from a disorganized capsule-ligamentous complex, and was removed en-bloc. The proximal end of the distal femoral shaft was transected to fresh edges and reamed proximally. The tibial prosthesis was removed, and the tibia was prepared using a tibial jig and block.

The long-stemmed customized femoral mega prosthesis was inserted after cementing the femoral and tibal canal with careful restoration of length, rotation, and version. The linea aspera was identified and an anti-lineal line, on the anterior femoral cortex, was used to ensure that we had the achieved proper rotation. The extensor apparatus was preserved during the procedure. The soft tissue tension and stability were assessed and found to be satisfactory and adequate. An intra-operative test of the range of movement was performed and full extension, and 100 degrees of flexion, was demonstrated. Patellar stability was assessed and found to be satisfactory in various degrees of flexion. A portovac drain was inserted and a sequential multi-layered wound closure was performed.

Post-operatively the patient was kept in bed with his right lower limb elevated on a pillow and passive physiotherapy was commended on the $3^{\text {rd }}$ post-operative day once pain allowed. One week post-operatively he began to mobilize to the chair alongside his hospital bed with the assistance of the physiotherapist. His wound healed well, and the skin clips were removed on the $10^{\text {th }}$ post-operative day. Two weeks post the surgery, once he could mobilize to the toilet and back albeit still with the assistance of a walker, he was discharged to the care of his family. At his 6-week follow-up appointment he was ambulant with a walking stick and was doing well. An Xray confirmed the distal femoral megaprosthesis in-situ (Figure 4). At his 1-year appointment he was independently ambulant although he had a slightly broad-based gait which we accounted for as due to his age. He was discharged for out-patient follow-up as needed.

\section{Discussion}

Besides the specific epidemiology of peri-prosthetic knee fractures, considerable attention is afforded to the risk factors for this complication in several papers. Regarding patient factors these include advanced age, osteoporosis, and medical conditions that predispose an individual to falling such as cardiac or neurological disease [16,17]. One paper notes the increasing mean age at which these fractures commonly occur, that while this was 67-years in 1986, it had increased to 78-years by 2010 [18]. With regards the site of the fracture itself namely femoral, tibial, or patellar, specific risk factors that are site specific are noted. While intra-operative peri-prosthetic fractures do occur, this paper limits itself to a discussion of peri-prosthetic fractures that occur post-operatively.

\section{Peri-prosthetic fractures of the Femur}

Distal femoral fractures are the most common site of periprosthetic knee fractures and the specific local risk factors here include

a. Anterior femoral notching which refers to deformation of the anterior femoral cortex during axial loading of the knee from overzealous resection of the anterior femur when the total knee replacement was performed,

b. Mismatching of the implant to the femoral cortex predisposing to loosening over time affecting stress distribution across the distal femur,

c. Implants that are rotationally constrained,

d. Revision surgery,

e. Delayed bony remodeling due to general medical conditions such as vascular compromise, 


\section{Orthopedics and Rheumatology Open Access Journal (OROAJ)}

f. Knee joint ankylosis, and

g. Poor bone quality $[19,20]$.

One paper quantifies the impact of femoral notching and reports that as little as a $3 \mathrm{~mm}$ anterior femoral cortical notch reduces the rotational torsional bone strength of the distal femur by as much as $30 \%$ [19]. Utilizing navigation to perform a total knee replacement is a further implicated risk factor as fracturing through the pins hole sites has been reported [21].

In terms of classification the Lewis and Rorabeck classification is widely used, specifically to classify peri-prosthetic distal femoral fractures, and as its basis uses femoral fracture displacement and the degree of fixation of the femoral prosthetic component. In this classification Type 1 fractures are undisplaced with a well fixed prosthesis and may be managed conservatively or by operative stabilization; Type 2 fractures have $>5 \mathrm{~mm}$ displacement / > 5 degrees of angulation with a well fixed prosthesis and require operative re-alignment and stabilization; and Type 3 fractures have a loose prosthetic component, irrespective of the fracture, and require revision of the prosthesis with/without femoral shaft replacement [22]. An alternative classification for these fractures, proposed by Kim et al, considers the bony volume in the distal fragment, the position and fixation status of the femoral prosthesis, and the reducibility of the fracture. In this classification Type 1 fractures have a well-aligned and stable femoral prosthesis, and subtypes of this include a Type $1 \mathrm{~A}$ where the fracture is reducible and amenable to conservative/operative management, and a Type $1 \mathrm{~B}$ when the fracture is irreducible and needs operative re-alignment. Type 2 fractures are characterized by a good distal bone stock however the femoral prosthesis is itself mal-aligned, or loose. This type is best managed by revision surgery. Lastly Type 3 fractures are characterized by comminution or insufficient distal bone stock and require a distal femoral replacement [23]. Considering our case, we consider the Kim Type 3 to best describe our patient's peri-prosthetic fracture as the almost completely absent distal femoral shaft and metaphysis necessitated a custommade replacement femoral shaft and knee prosthesis.

\section{A Conservative}

Non-displaced femoral fractures with an intact prosthesis can be managed by simple immobilization however several studies recognize a high incidence of medical complications and non-union in immobilized elderly patients. For this reason, these same studies recommend operative fixation rather than conservative immobilization, which allows early mobilization and the preservation of functional range of motion $[19,24]$.

\section{Operative stabilization}

\section{Intra-medullary nailing}

Fundamentally all stabilization devices can be classified as either intra or extra-medullary. Retro-grade intramedullary femoral nailing is the technique of choice, in fractures that are aligned or able to be aligned with a fixed prosthesis, however several requirements need to be met for this technique to be employed. These include a favorable total knee prosthetic design with a box that is design compatible with nail insertion, the absence of a stem on the prosthesis that if present would preclude nail insertion, and additional hardware proximal to the knee that would interfere with the nail. A concomitant ipsilateral total hip replacement with a femoral stem is a relative contra-indication as the interval between the stem, and the intra-medullary nail, would create a stress riser predisposed to fracturing itself [25].

The minimal soft tissue stripping inherent to the plating techniques makes retrograde intra-medullary nailing an attractive option. In cases where the prosthesis is well-aligned and fixed, and the fracture reducible, several studies have shown that the insertion of a retrograde intra-medullary nail, to address a peri-prosthetic femur fracture, results in an excellent union rate and a high degree of functional outcome. In these same studies an intra-medullary nail design that incorporates proximal and distal interlocking screws offers superior resistance to axial compression and torsional forces making it the specific implant of choice $[17,26]$. Further important points regarding this technique are that the intramedullary nail must firstly be long enough to reach the level of the lesser trochanter as the necessary passage through the femoral isthmus is needed to prevent toggling and thereby prevent what is terms in one study as the windscreenwiper effect [27]. A second important point is that in cases where there is valgus malalignment of the distal fragment post reduction, a blocking screw can be inserted to guide retrograde nail insertion [28]. The use of flexible intra-medullary nails, as was advocated in the past, are no longer advised except as an option in minimally displaced fractures. Their inherently poor resistance to compression and rotational loading is now recognized to lead to a high incidence of limb shortening and malunion making their use non advisable [10].

\section{Plating}

Locking plates are considered at least an equal alternative to retrograde intra-medullary nailing, and both have been demonstrated to be significantly superior fixation techniques to non-locking plates $[29,30]$. Locking plates have an additional unique advantage over retrograde intra-medullary nailing in that they can be used in cases of exceptionally poor bone stock [31]. Comminution of the medial femur is a special consideration when plating is being considered, and in these cases either dual plating or retrograde nailing are both options to prevent the development of a varus deformity secondary to medial femoral collapse [32]. One cohort study showed that while locking plates demonstrated a $96 \%$ union rate, this frequently required restricted weight bearing for up to 3-monthand may take as long as 6 months to occur [33]. The newer poly-axial screw locking plates have an additional advantage in that stability can be achieved without interfering with the fixed femoral prosthesis or interface below [34]. 


\section{Revision total knee arthroplasty and Distal femoral mega-prosthesis}

As soon as a loose implant is encountered, retrograde intramedullary nailing and plating are no longer options, and total revision of the implant incorporating a cemented long stem femoral prosthesis becomes mandatory [35]. In the setting of a loose prosthesis with poor bone stock, either allograft must be used to reconstruct the distal femur to allow a revision total knee arthroplasty, or replacement of the distal femoral shaft and femur with a distal femoral mega-prosthesis must be performed [36,37]. Despite the traditional thinking that a distal femoral replacement would incur a high complication rate in the elderly, one study that compared this to standard revision total knee arthroplasty and allograft implant composites, found that the distal femoral replacement group did not incur a higher complication rate and in fact demonstrated the shortest duration of surgery, least intraoperative blood loss, and fastest recovery time [38]. Another study that considered 5 patients, who underwent distal femoral replacement with a mega-prosthesis due to poor distal bone stock, reported early ambulation and a 100-degree range of motion in 4/5 (80\%) subjects [39].

\section{Peri-prosthetic fractures of the Tibia}

Most post-operative peri-prosthetic tibial fractures are related to acute trauma however stress fracturing has been reported [40]. More often that in the more commonly occurring periprosthetic fractures of the femur, is an association with implant loosening and joint instability [10]. In terms of classifying these fractures the Mayo classification is widely used and incorporates 1. Whether the fracture occurred intra-operatively / postoperatively, 2. Fracture location, and 3. Implant stability. Utilizing these variables, Type 1 fractures comprise a depression/split that involves the tibial plateau and extends to the implant interface; Type 2 fractures refers to those occurring adjacent to the implant stem; Type 3 fractures are diaphyseal fractures occurring distal to the prosthesis; and Type IV fractures are avulsion fractures of the tibial tubercle. A sub-classification of Types 1-3 affords each into a $\mathrm{A}, \mathrm{B}$, or $\mathrm{C}$ sub-category depending on whether the prosthesis is fixed (A), loose (B), or occurred intra-operatively (C) [41]. In terms of management conservative treatment is an option, for undisplaced / reducible fractures where the implant remains fixed, however complications due to prolonged immobilization and limited weight bearing in the elderly are problematic . Open reduction and plating are advocated to allow early mobilization and to reduce displaced fractures, but again require the implant to be fixed. Revision arthroplasty with long stemmed cemented tibial implants are advocated when there is implant loosening [42]. In those that require operative intervention the pre-existing tibial component impedes the use of an intra-medullary nail. Hence, with regards peri-prosthetic fractures of the tibia, either locking plates in fractures where the tibial prosthesis is fixed, or a long stemmed tibial prosthesis that transverse the fracture line when the implant is loose, are the two primary surgical interventions $[41,42]$.

\section{Peri-prosthetic fractures of the Patella}

Several studies confirm the incidence of peri-prosthetic fractures of the patella to be strongly influenced by whether the patella has been resurfaced or not. In the patella-resurfaced knee the incidence is reported to be as high as $21 \%$, while in the nonresurfaced patella the incidence is reported to be as low as $0.05 \%$ $[43,44]$. The commonest mechanism of injury in peri-prosthetic patella fractures is repetitive stress trauma secondary to technical re-surfacing errors and subsequent malalignment [44-46]. The most common classification system for these fractures is that of Ortiguera and Berry which is based on three parameters, 1. Extensor mechanism integrity, 2. Fixation of the patella, and 3. Quality of residual bone stock.

Using this classification system, Type 1 fractures comprise a fracture occurring in the context of a fixed prosthesis and intact extensor mechanism. These fractures are best managed conservatively with immobilization in full extension and then graded flexion once bony union has occurred. Conservative management of Type 1 fractures is reported to have a good outcome in over $96 \%$ of cases [44]. Type 2 fractures are those that occur in the context of a fixed prosthesis however have a disrupted extensor mechanism. Here the amount of fracture displacement, and thickness of the patellar bone stock, dictate management. Fractures with $<2 \mathrm{~mm}$ of displacement may be managed conservatively with immobilization, while those that demonstrate $>2 \mathrm{~mm}$ of displacement need surgical intervention, either in the form of revision surgery if there is $>10 \mathrm{~mm}$ of patellar bone stock, or excision arthroplasty if there is $<10 \mathrm{~mm}$ of patellar bone stock. Type 3a fractures refer to fractures occurring in the context of a loose prosthesis, and reasonable $(>10 \mathrm{~mm})$ patellar bone stock, and are best managed by revision surgery. Type $3 \mathrm{~b}$ fractures are those that occur in the context of a loose prosthesis with poor $(<10 \mathrm{~mm})$ patellar bone stock, and these are best managed by excision arthroplasty [42].

\section{Conclusion}

The globally increasing incidence of total knee replacement surgeries is underpinning the parallel increasing incidence in peri-prosthetic knee fractures. These fractures are difficult to manage, and complications are common. Even in fractures that heal with satisfactory alignment a significant proportion of these patients will need long-term assistance with ambulating. As such any orthopedic surgeon who performs knee arthroplasty surgery must have a sound understanding, and working algorithm, for how he/she manages peri-prosthetic knee fractures. The extensive literature on the subject is testament to the high and increasing incidence of this problem. Our case report provides an extreme example of a recurrent peri-prosthetic distal femur fracture that, due to almost non-existent distal bone stock, required a 
custom-made distal femoral mega-prosthesis. It is hoped that our case report and review of the subject will assist orthopedic surgeons who perform knee replacement surgeries and will, in all probability, need to manage this specific group of patients.

\section{Funding}

This research did not receive any specific grant from funding agencies in the public, commercial, or not-for-profit sectors.

\section{Conflicts of Interest Statement}

None of the authors listed above have any financial nor personal relationships with other people, or organizations, that could inappropriately influence (bias) their work, all within 3 years of the beginning the work submitted.

\section{References}

1. Kurtz S, Ong K, Lau E, Mowat F, Halpern M (2007) Projections of primary and revision hip and knee arthroplasty in the United States from 2005 to 2030. J Bone Joint Surg Am 89(4): 780-785.

2. Han HS, Oh KW, Kang SB (2009) Retrograde intramedullary nailing for periprosthetic supracondylar fractures of the femur after total knee arthroplasty. Clin Orthop Surg 1(4): 201-206.

3. Kim KI, Egol KA, Hozack WJ, Parvizi J (2006) Periprosthetic fractures after total knee arthroplasties. Clin Orthop Relat Res 446: 167-175.

4. Ricci WM (2015) Periprosthetic femur fractures. J Orthop Trauma 29(3): 130-137.

5. Parvizi J, Jain N, Schmidt AH (2008) Periprosthetic knee fractures. J Orthop Trauma 22(9): 663-671.

6. Agarwal S, Sharma RK, Jain JK (2014) Periprosthetic fractures after total knee arthroplasty. J Orthop Surg 22(1): 24-29.

7. Haller JM, Kubiak EN, Spiguel A, Gardner MJ, Horwitz DS (2012) Intramedullary nailing of tibial shaft fractures distal to total knee arthroplasty. J Orthop Trauma 28(12): e296-300.

8. Chalidis BE, Tsiridis E, Tragas AA, Stavrou Z, Giannoudis PV (2007) Management of periprosthetic patellar fractures. A systematic review of literature. Injury 38(6): 714-724.

9. Herrera DA, Kregor PJ, Cole PA, Levy BA, Jönsson A, et al. (2008) Treatment of acute distal femur fractures above a total knee arthroplasty: systematic review of 415 cases (1981-2006). Acta Orthop 79(1): 22-27.

10. Dennis DA (2001) Periprosthetic fractures following total knee arthroplasty. J Bone Joint Surg Am 83: 120-130.

11. Rorabeck CH, Taylor JW (1999) Periprosthetic fractures of the femur complicating total knee arthroplasty. Orthop Clin North Am 30(2): 265-277.

12. Gondalia V, Choi DH, Lee SC, Nam CH, Hwang BH, et al. (2014) Periprosthetic supracondylar femoral fractures following total knee arthroplasty: clinical comparison and related complications of the femur plate system and retrograde-inserted supracondylar nail. J Orthop Traumatol 15(3): 201-207.

13. Hoffmann MF, Jones CB, Sietsema DL, Koenig SJ, Tornetta P (2012) Outcome of periprosthetic distal femoral fractures following knee arthroplasty. Injury 43(7): 1084-1089.
14. Chen F, Mont MA, Bachner RS (1994) Management of ipsilateral supracondylar femur fractures following total knee arthroplasty. J Arthroplasty 9(5): 521-526.

15. Cordeiro EN, Costa RC, Carazzato JG, Silva Jdos S (1990) Periprosthetic fractures in patients with total knee arthroplasties. Clin Orthop Relat Res 252: 182-189.

16. Herrera DA, Kregor PJ, Cole PA, Levy BA, Jönsson A, et al. (2008) Treatment of acute distal femur fractures above a total knee arthroplasty: systematic review of 415 cases (1981-2006). Acta Orthop 79(1): 22-27.

17. Yoo JD, Kim NK (2015) Periprosthetic fractures following total knee arthroplasty. Knee Surg Relat Res 27(1): 1-9.

18. Gondalia V, Choi DH, Lee SC, Nam CH, Hwang BH, et al. (2014) Periprosthetic supracondylar femoral fractures following total knee arthroplasty: clinical comparison and related complications of the femur plate system and retrograde-inserted supracondylar nail. J Orthop Traumatol 15(3): 201-207.

19. Culp RW, Schmidt RG, Hanks G, Mak A, Esterhai JL Jr, et al. (1987) Supracondylar fracture of the femur following prosthetic knee arthroplasty. Clin Orthop Relat Res 222: 212-222.

20. Chen F, Mont MA, Bachner RS (1994) Management of ipsilateral supracondylar femur fractures following total knee arthroplasty. J Arthroplasty 9(5): 521-526.

21. Li CH, Chen TH, Su YP, Shao PC, Lee KS, et al. (2008) Peri -prosthetic femoral supracondylar fracture after total knee arthroplasty with navigation system. J Arthroplasty 23(2): 304-307.

22. Rorabeck CH, Taylor JW (1999) Classification of periprosthetic fractures complicating total knee arthroplasty. Orthop Clin North Am 30: 209-214.

23. Kim K-I, Egol KA, Hozack WJ, Parvizi J (2006) Periprosthetic fractures after total knee arthroplasties. Clin Orthop Relat Res 446: 167-175.

24. Delport PH, Van Audekercke R, Martens M, Muller JC (1984) Conservative treatment of ipsilateral supracondylar femoral fracture after total knee arthroplasty. J Trauma 24: 846-849.

25. Whitehouse M, Mehendale S (2014) Periprosthetic fractures around the knee: current concepts and advances in management. Curr Rev Musculoskelet Med 7(2):136-144.

26. Murrell GA, NunleyJA (1995) Interlocked supracondylar intramedullary nails for supracondylar fractures after total knee arthroplasty: a new treatment method. J Arthroplasty 10(1): 37-42.

27. Lee SS, Lim SJ, Moon YW, Seo JG (2014) Outcomes of long retrograde intramedullary nailing for periprosthetic supracondylar femoral fractures following total knee arthroplasty. Arch Orthop Trauma Surg 134(1): 47-52.

28. Jabczenski FF, Crawford M (1995) Retrograde intramedullary nailing of supracondylar femur fractures above total knee arthroplasty: a preliminary report of four cases. J Arthroplasty 10(1): 95-101.

29. DiGioia AM $3^{\text {rd }}$, Rubash HE (1991) Periprosthetic fractures of the femur after total knee arthroplasty. A literature review and treatment algorithm. Clin Orthop Relat Res 271: 135-142.

30. Frigg R, Appenzeller A, Christensen R, Frenk A, Gilbert S, et al. (2001) The development of the distal femur Less Invasive Stabilization System (LISS). Injury 32(3): 24-31.

31. Althausen PL, Lee MA, Finkemeier C (2003) Operative stabilization of supracondylar femur fractures above total knee arthroplasty: a comparison of four treatment methods. J Arthroplasty 18(7): 834-839. 
32. Gurava Reddy V, Krishna Mootha A, Chiranjeevi T, Kantesaria P, Kumar Ramireddy V, et al. (2011) Bilateral symmetrical periprosthetic (mirror) fractures of knee fixed with dual plating technique. Int J Surg Case Rep 2: 175-177.

33. Hassan S, Swamy GN, Malhotra R, Badhe NP. Periprosthetic fracture of the distal femur after total knee arthroplasty; prevalence and outcomes following treatment. J Bone Joint Surg (Br). 2012;94(24):6.

34. Ruchholtz S, Tomás J, Gebhard F, Larsen MS (2013) Periprosthetic fractures around the knee-the best way of treatment. Euro Orthop Traumatol 4(2): 93-102.

35. Srinivasan K, Macdonald DA, Tzioupis CC, giannoudis PV (2005) Role of long stem revision knee prosthesis in periprosthetic and complex distal femoral fractures: a review of eight patients. Injury 36(9): 10941102.

36. Kassab M, Zalzal P, Azores M (2004) Management of periprosthetic femoral fractures after total knee arthroplasty using a distal femoral allograft. J Arthroplasty 19(3): 361-368.

37. Backstein D, Safir 0, Gross A (2006) Management of bone loss: structural grafts in revision total knee arthroplasty. Clin Orthop Relat Res 446: 104-112.

38. Saidi K, Ben-Lulu 0, Tsuji M, Safir O, Gross AE, et al. (2014) Supracondylar periprosthetic fractures of the knee in the elderly patients: a comparison of treatment using Allograft-Implant Composites, Standard Revision Components, Distal Femoral Replacement Prosthesis. J Arthroplasty 29: $110-114$
39. Freedman EL, Hak DJ, Johnson EE, Eckardt JJ (1995) Total knee replacement including a modular distal femoral component in elderly patients with acute fracture or nonunion. J Orthop Trauma 9(3): 231237.

40. Rand JA, Coventry MB (1980) Stress fractures after total knee arthroplasty. J Bone Joint Surg Am. 62(2): 226-233.

41. Felix NA, Stuart MJ, Hanssen AD (1997) Periprosthetic fractures of the tibia associated with total knee arthroplasty. Clin Orthop Relat Res 345: 113-124.

42. Sarmah SS, Patel S, Reading G, El-Husseiny M, Douglas S, et al. (2012) Periprosthetic fractures around total knee arthroplasty. Ann R Coll Surg Engl 94(5): 302-307.

43. Goldberg VM, Figgie HE $3^{\text {rd }}$, Inglis AE, Figgie MP, Sobel M, Kelly M, et al. (1988) Patellar fracture type and prognosis in condylar total knee arthroplasty. Clin Orthop Relat Res 236: 115-122.

44. Ortiguera CJ, Berry DJ (2002) Patellar fracture after total knee arthroplasty. J Bone Joint Surg Am 84(4): 532-540.

45. Chalidis BE, Tsiridis E, Tragas AA, Stavrou Z, Giannoudis PV (2007) Management of periprosthetic patellar fractures. A systematic review of literature. Injury 38(6): 714-724.

46. Yoo JD, Kim NK (2015) Periprosthetic fractures following total knee arthroplasty. Knee Surg Relat Res 27(1): 1-9.

\section{Your next submission with Juniper Publishers will reach you the below assets}

- Quality Editorial service

- Swift Peer Review

- Reprints availability

- E-prints Service

- Manuscript Podcast for convenient understanding

- Global attainment for your research

- Manuscript accessibility in different formats

( Pdf, E-pub, Full Text, Audio)

- Unceasing customer service

Track the below URL for one-step submission https://juniperpublishers.com/online-submission.php 